\title{
Frequency and Causes of False Negative Diffusion- Weighted Imaging in Acute Ischemic Stroke
}

\author{
Burç Esra Şahin ${ }^{1}(\underline{I D})$ \\ ${ }^{1}$ Department of Neurology, Faculty of Medicine, Kırşehir Ahi Evran University, Kırsehir, Turkey
}

Copyright@ Author(s) - Available online at https://dergipark.org.tr/en/pub/mbsjohs Content of this journal is licensed under a Creative Commons Attribution-NonCommercial 4.0 International License,

Received: 22 April 2021, Accepted: 10 August 2021, Published online: 31 August 2021

(C) Ordu University Institute of Health Sciences, Turkey, 2021

\begin{abstract}
Objective: The high sensitivity of diffusion-weighted magnetic resonance imaging (DWI MRI) has led to its frequent use in the diagnosis of acute ischemic stroke (AIS). However, false negative DWI MRI results have been obtained for some patients diagnosed with stroke, which led us to initiate this study. Our aim was to determine the prevalence of false negative DWI MRI scans and prevent the clinician from making a late diagnosis or misdiagnosis by relying on MRI results only.

Methods: In a retrospective file screening conducted between February 2017- February 2019, after the patients hospitalized with a diagnosis of ischemic stroke who couldn't have an MRI or who were diagnosed with transient ischemic attack were excluded, the frequency of patients with a normal initial cranial DW MRI scan whose follow up scans revealed acute diffusion restriction was identified, and vascular anatomical localization of stroke was classified according to OCSP (Oxfordshire Community Stroke Project).

Results: Of 235 patients admitted to our clinic with a diagnosis of ischemic stroke, 21 couldn't have a DWI MRI, and of 214 stroke patients who had a DWI MRI, 23 were admitted with a transient ischemic stroke attack. Of the remaining 191 patients, 14 had initially negative DWI MRI images but their clinical findings lasted longer than $24 \mathrm{hrs}$ so they had a follow up MRI, which revealed an ischemic lesion in brain diffusion. In our clinic, the percentage of false negative diffusion MR images was 7.3\% (14/191). The distribution of ischemia in the aforementioned 14 patients was as follows: 6 patients with posterior circulation ischemia (POCI), including 4 in brain stem and 2 in cerebellum, 2 patients with lacunar stroke (LACI), 5 patients with partial anterior circulation ischemia (PACI) and 1 patient with total anterior circulation ischemia (TACI).When the time of symptom onset was questioned, data could be derived from only 8 patients' files, and DWI MR images were obtained within the first 6 hours according to the onset of the symptoms.

Conclusion: In acute stroke patients, if symptoms of the patient are consistent with stroke during physical examination, the diagnosis of stroke should not be automatically ruled out even if brain DWI MRI is negative. The decision of urgent thrombolytic or endovascular intervention that can be taken for eligible patients should not be overlooked based on false negative DWI MRI findings. With this study, we aim to help clinicians avoid misdiagnosis or delays in diagnosis.
\end{abstract}

Key words: Diffusion magnetic resonance, Imaging, Stroke, False negative

Suggested Citation: Sahin BE. Frequency and Causes of False Negative Diffusion-Weighted Imaging in Acute Ischemic Stroke Mid Blac Sea Journal of Health Sci, 2021;7(2):230-235

Address for correspondence/reprints:

Telephone number: +90 (506) 5993494

Burc Esra Sahin

E-mail: burcesrasahin@gmail.com 


\section{Introduction}

Early and rapid thrombolytic or endovascular therapy has been shown to increase recovery in the long term. Acute ischemic stroke (AIS) is a clinical diagnosis based on sudden focal neurological deficits $(1,2)$.

Accordingly, the inclusion criteria for the landmark 1995 National Institute of Neurological Disorders and Stroke study of IV tissue plasminogen activator (tPA) for AIS included a neurological deficit measurable on NIH Stroke Scale (3). The guide published by the American Academy of Neurology (AAN) in 2010 says "DWI needs to be done for the most accurate diagnosis of acute ischemic stroke"(4).

Recently, there has been a significant increase in the use of brain MRI in the evaluation of patients with AIS (5). DWI MRI has a significantly higher sensitivity $(96.7 \%)$ and specificity $(93.4 \%)$ than CT in detecting acute ischemia (6). It's recommended that brain CT, one of the imaging methods, is used as soon as possible to rule out intracranial hemorrhage, tumor or abscess contraindicated to thrombolysis (7-9). Non-contrast CT is an easily accessible, versatile and rapid procedure, making it a better choice than MRI in imaging of acute ischemia when considering thrombolytic therapy, and it's also cost-effective (10).

With increasing DWI MRI use over the years, evidence showing that it cannot identify AIS albeit in a small group of patients has emerged. Causes of diffusion-negative cases are classified into 3 categories. The first group is posterior circulatory system ischemia, the second group is minor ischemia (especially in the brain stem), and the third group consists of those who present in the hyperacute period and may be overlooked in DWI MRI (11-17).

The conflicts between clinical and radiological findings in AIS patients forced us to investigate the reasons for such conflicts. We aimed to detect false negative DWI MRI prevalence in our clinic, bring to the attention of the clinicians that there may also be diffusion negative AIS cases and help them avoid misdiagnosis or delays in diagnosis by relying on MRI results only.

\section{Methods}

After consent of the local ethics committee was obtained, the patients admitted with a diagnosis of ischemic stroke to the Neurology Clinic of Ahi Evran University in Kirsehir between February 2017-
February 2019 were included in the study by retrospective file screening. After the patients who couldn't have an MRI or who were diagnosed with transient ischemic attack were excluded, the frequency of patients with a normal initial DWI MR image whose follow up images revealed acute diffusion restriction was identified, and these patients were classified according to OCSP (Oxfordshire Community Stroke Project). MR images of the study patients with a negative DWI MRI were assessed by a radiologist who has no knowledge of their clinical presentation and 2 neurologists who have knowledge of their clinical examinations. In our hospital, DWI sequences; a 5-mm thick cross-section area, b value 1000 s. mm- ${ }^{2}$, 1.5 Tesla MR are used.

Statistical Analysis: Statistical analyzes of the study were performed using Statistical Package for Social Sciences version 25.0 software for Windows (IBM SPSS Statistics for Windows, Version 25.0. Armonk, NY: IBM Corp., USA). In the study, descriptive statistics are given as mean $\overline{\text { standard }}$ deviation and $\mathrm{n}(\%)$.

\section{Results}

Of 235 patients admitted to our clinic with a diagnosis of ischemic stroke, 21 couldn't have a DWI MRI, and of 214 AIS patients who had a DWI MRI, 23 were admitted with a transient ischemic stroke attack. Of the remaining 191 patients, 14 had initially negative DWI MRI images but their clinical findings lasted longer than 24 hours, so they had a follow up MRI, which revealed an ischemic lesion in brain diffusion (Figure 1).

In our clinic, the percentage of false negative diffusion MR images was $7.3 \%$ (14/191). The distribution of ischemia in the aforementioned 14 patients was as follows: 6 patients (42.8\%) with posterior circulation ischemia (POCI), including 4 in brain stem and 2 in cerebellum. 2 patients (14.2\%) with lacunar stroke (LACI), 5 patients (35.7\%) with partial anterior circulation ischemia (PACI) and 1 patient $(7.1 \%)$ with total anterior circulation ischemia (TACI) (Figure 2). When the time of symptom onset was questioned, data could be derived from only 8 patients' files, and DWI MRI were obtained within the first 6 hours according to the onset of the symptoms. 


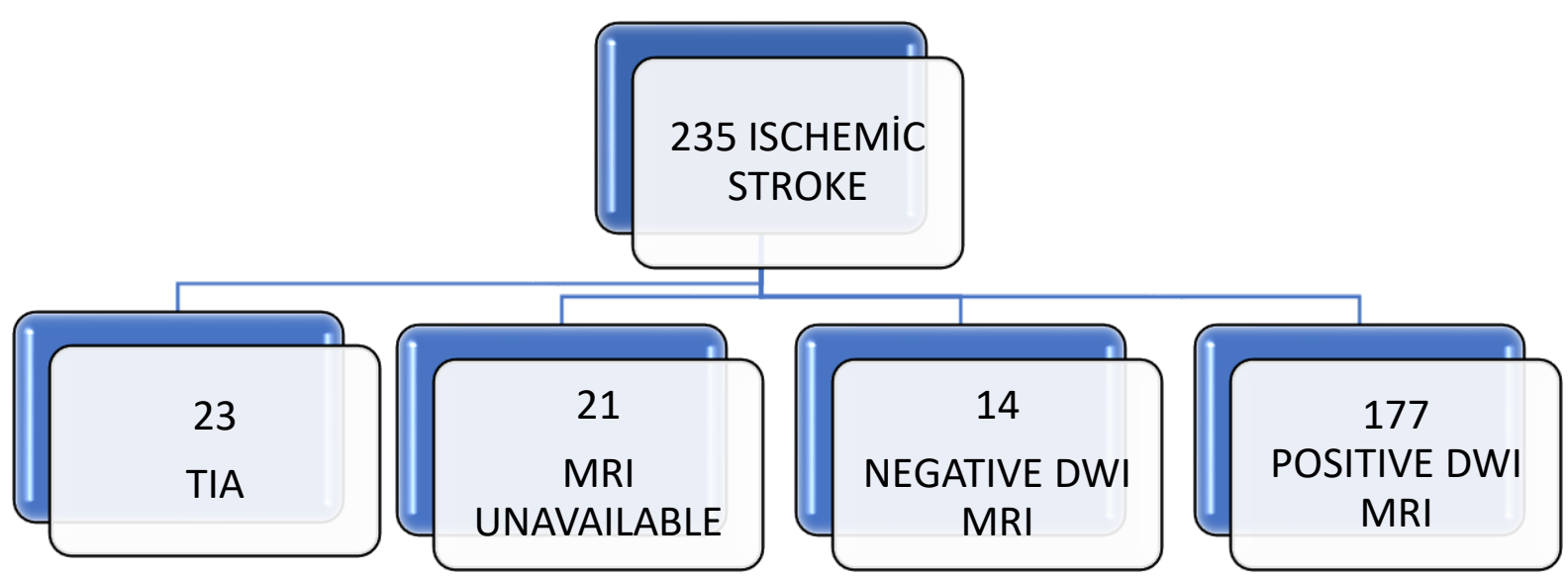

Figure 1. Distribution of Ischemic Stroke Patients

\section{OCSP}

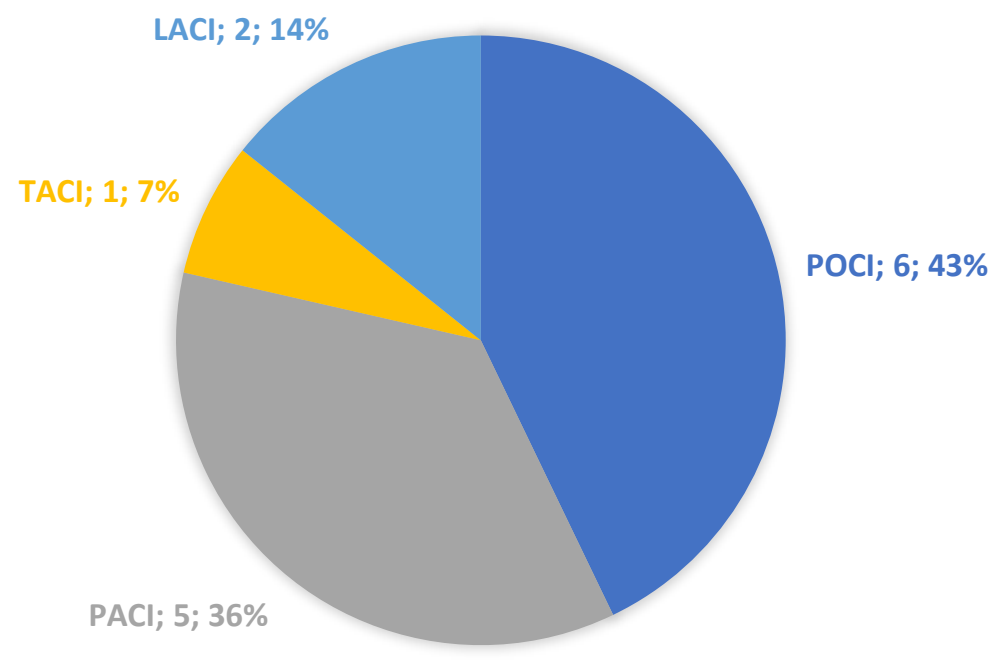

Figure 2 Clinical Classification According to OCSP (Oxfordshire Community Stroke Project)

\section{Discussion}

MRI diffusion negativity is an expected finding in patients presenting with transient ischemic stroke and stroke-like clinical presentation (seizures, migraine, vertigo). However, although examination findings of such patients were consistent with the clinical symptoms of stroke, their DWI MRI at admission didn't reveal diffusion restriction. Their follow up DWI MRI revealed diffusion limitation, which forced us to investigate the reasons for this observation and question the diagnostic criteria again.

Among stroke and TIA admissions $15.7 \%$ of cases had initial DWI-negative scans at the beginning of follow-up (18). Edlow et al.'s review reported a false negative DWI MRI percentage of $6.8 \%$ 17.4) (9). We found a similar false negative DWI MRI percentage in AIS patients in our clinic $(7.4 \%$ (14/191)).

Only 2 studies evaluated the percentage of negative DWI MRI by comparing the DWI MRI scans performed in the first 6 hours of stroke with the scans performed 6 hours later $(19,20)$. In the first study, the rate of negative DWI MRI scans performed in the first 6 hours of stroke onset was 5.9\% (2 of 34 patients) and $13.7 \%$ (16 of 117 patients) performed 6 hours later (19). In the second study, the rate of negative DWI MRI scans performed in the first 6 hours of stroke onset time was $14.3 \%$ (2 of 14 
patients) and 2.2\% (1 of 45 patients) performed 6 hours after the stroke onset time (20). Some studies made comments about DWI MRI time and the rate of negativity although they did not use the 6-hour threshold value. Chalela et al. found that diffusion negativity was strongly associated with DWI MRI scan (OR (95\% CI) $5.8(2.3-14.9))$ performed in the first 3 hours(21). Similarly, Simonsen et al. reported that DWI-negative AIS patients underwent DWI MRI sooner after symptom onset than DWI-positive AIS patients, an average of 109 minutes versus 120 minutes, respectively $(\mathrm{p}<0.05) \quad(22)$. All of our patients whose records were accessible presented within the first 6 hours and diffusion positivity was detected within 24 hours in repeated scans. But data about the duration could be obtained from only 8 patients' files.

$10 \%$ of AIS cases are brainstem ischemia and observed in pons, medulla oblongata and the mesencephalon. The main arterial supply of the brain stem is provided by the vertebral artery, anterior spinal artery, posterior inferior cerebellar artery and posterior cerebral artery. A study by Phillippe et al. reported that of 155 brainstem ischemia patients, ischemia was localized in mesencephalon in 12 patients, pons in 115 patients and medulla oblongata in 31 patients. Standard axial $(5 \mathrm{~mm})$ and thin-section coronal (3mm) DWI MRIs were performed for each patient. $3(1.9 \%)$ patients had ischemia in the medulla oblongata, which could only be viewed in thinsection coronal (3mm) DWI MRI. In comparison, 35 $(22.6 \%)$ patients had ischemic lesions, which were more prominent with coronal imaging than axial imaging and found to be significantly smaller (0.06 $\mathrm{cm} 3, \mathrm{p}<0.001)$ (23). If the lesion is small, highresolution DWI MRI combining thinner axial and coronal sections increases sensitivity in identifying these lesions $(13,24)$.

In a meta-analysis of 5 studies in the literature, the odds ratio (OR) of posterior circulatory ischemia relative to the anterior system was found to be 5.1 (9). However, in our study, the ratio of posterior system to anterior system was $8 / 6$, whereas Bulut et al. found an equal ratio (25).

There's limited number of studies investigating the relationship between DWI MRI false negativity and small stroke volume. One study reported an average infarct size \pm standard deviation of $0.19 \pm 0.16 \mathrm{~cm}^{3}$ (range $0.05-0.5 \mathrm{~cm}^{3}$ ) (26).

False negative conditions can occur especially in the vertebrobasillary region and in lacunar ischemia due to its small size or anisotropic sensitivity artefacts $(6,13,26)$. 3.0 Tesla MRI is more advantageous due to higher signal to noise ratio (SNR) but has larger sensitivity artifacts $(27,28)$. Despite the high SNR of 3.0 T MRI, DWI contrast was $18 \%$ lower compared to $1.5 \mathrm{~T}$ MRI, which surprisingly led to the finding that diffusion changes in acute AIS are less visible in 3 T MRI, which also has lesser diagnostic power. In the diagnosis of AIS, the sensitivity of $1.5 \mathrm{~T}$ MR DWI was $99.1 \%$ and that of $3.0 \mathrm{~T}$ MR was $92.5 \%$, and the specificity decreased from $97.8 \%$ at $1.5 \mathrm{~T}$ to $84.1 \%$ at 3.0 T (6).

In addition, as the magnetic force area increased, image distortion artifacts became heavier $(27,29,30)$. These bright artifacts may mask some early ischemic changes, especially in the skull base. This is particularly worrisome at the very early stage of stroke (in the first 2 hours) when there are certain DWI findings. In other words, false negativity increases with early imaging and 3 T MRI (6).

After it was understood that there might be negative DWI MRI scans, various techniques were tried to increase the sensitivity of MRI. Primary approaches included perfusion-weighted MRI (PWI) scans incorporating gadolinium-based and arterialspin-labeled techniques or increasing the spatial resolution of DWI MRI sequences, reducing geometric distortion or a second coronal section scan from the posterior fossa were applied (9). In another study compared imaging methods to help the clinical detection of cerebral infarction(DWI was $97 \%$ (33/34) , DTI was $94 \%$ (32/34), SWI was $88 \%$ (30/34), T2WI was $79 \%(27 / 34)$ ) and found that combined imaging is better than single imaging (31).

Addition of a PWI scan may increase DWI sensivity from $92 \%$ to $97.5 \%$ (22). However, its disadvantages include time and contrast agent side effects.

To increase the DWI sensitivity, the 'b value', which is a value that reflects the strength and duration of the diffusion gradients applied to water molecules in the brain, can be increased. It is argued that the increase in the $b$ value $\left(1000 \mathrm{~s} . \mathrm{mm}^{2}\right)$ in diffusionweighted scans may increase the sensitivity to minor ischemia $(9,32,33)$.

Currently, neither complementary MRI sequences such as PWI nor high b-value DWI techniques have gained wide recognition in the clinical evaluation of patients with AIS (9).

The study has some limitations. First, it is necessary to validate these findings with a larger cohort to reach a more definitive conclusion. Second, this study was retrospective. Third, MRI's were not always performed by the same technician. 


\section{Conclusion}

These results show that in AIS cases, if the patient's symptoms are consistent with stroke on examination, the diagnosis of stroke should not be immediately excluded, even if DWI MRI is negative. The decision of urgent thrombolytic or endovascular intervention that can be taken for eligible patients should not be overlooked based on false negative DWI MRI findings. We recommend that the clinician should rule out conditions that cause contraindication to brain CT in all patients with suspected AIS during examination and assess such patients in terms of the suitability of IV tissue plasminogen activator treatment without delay.

Future studies should focus on methods to increase DWI MRI sensitivity, the hour at which diffusion-negative patients develop diffusion positivity, and its relationship with the volume of ischemia.

Ethics Committee Approval: This study was approved by the Ethical Committee of Kirsehir Ahi Evran University School of Medicine number of 2019-04/56.

Peer-review: Externally peer-reviewed.

Author Contributions:

Concept, Design, Literature search, Data Collection and Processing, Analysis or Interpretation, Writing - BES

Conflict of Interest: No conflict of interest was declared by the authors.

Financial Disclosure: The author declared that this study hasn't received no financial support.

\section{References}

1. Saver JL, Fonarow GC, Smith EE, Reeves MJ, Grau-Sepulveda MV, Pan W, et al. Time to treatment with intravenous tissue plasminogen activator and outcome from acute ischemic stroke. JAMA. 2013;309(23):2480-8.

2. Goyal M, Menon BK, van Zwam WH, Dippel DW, Mitchell PJ, Demchuk AM, et al. Endovascular thrombectomy after large-vessel ischaemic stroke: a meta-analysis of individual patient data from five randomised trials. Lancet. 2016;387(10029):1723-31.

3. National Institute of Neurological D, Stroke rt PASSG. Tissue plasminogen activator for acute ischemic stroke. N Engl J Med. 1995;333(24):1581-7.
4. Schellinger PD, Bryan RN, Caplan LR, Detre JA, Edelman RR, Jaigobin C, et al. Evidence-based guideline: The role of diffusion and perfusion MRI for the diagnosis of acute ischemic stroke: report of the Therapeutics and Technology Assessment Subcommittee of the American Academy of Neurology. Neurology. 2010;75(2):177-85.

5. Burke JF, Kerber KA, Iwashyna TJ, Morgenstern LB. Wide variation and rising utilization of stroke magnetic resonance imaging: data from 11 states. Ann Neurol. 2012;71(2):179-85.

6. Rosso C, Drier A, Lacroix D, Mutlu G, Pires C, Lehericy $\mathrm{S}$, et al. Diffusion-weighted MRI in acute stroke within the first 6 hours: 1.5 or 3.0 Tesla? Neurology. 2010;74(24):1946-53.

7. Brazzelli M, Sandercock PA, Chappell FM, Celani MG, Righetti E, Arestis N, et al. Magnetic resonance imaging versus computed tomography for detection of acute vascular lesions in patients presenting with stroke symptoms. Cochrane Database Syst Rev. 2009(4):CD007424.

8. National Collaborating Centre for Chronic C. National Institute for Health and Clinical Excellence: Guidance. Stroke: National Clinical Guideline for Diagnosis and Initial Management of Acute Stroke and Transient Ischaemic Attack (TIA). National Institute for Health and Clinical Excellence: Guidance. London: Royal College of Physicians (UK) Copyright (C) 2008, Royal College of Physicians of London.; 2008.

9. Edlow BL, Hurwitz S, Edlow JA. Diagnosis of DWI-negative acute ischemic stroke: A metaanalysis. Neurology. 2017;89(3):256-62.

10.Wardlaw JM, Seymour J, Cairns J, Keir S, Lewis $S$, Sandercock P. Immediate computed tomography scanning of acute stroke is costeffective and improves quality of life. Stroke. 2004;35(11):2477-83.

11.Kattah JC, Talkad AV, Wang DZ, Hsieh YH, Newman-Toker DE. HINTS to diagnose stroke in the acute vestibular syndrome: three-step bedside oculomotor examination more sensitive than early MRI diffusion-weighted imaging. Stroke. 2009;40(11):3504-10.

12.Ay H, Buonanno FS, Rordorf G, Schaefer PW, Schwamm LH, Wu O, et al. Normal diffusionweighted MRI during stroke-like deficits. Neurology. 1999;52(9):1784-92.

13. Sylaja PN, Coutts SB, Krol A, Hill MD, Demchuk AM, Group VS. When to expect negative diffusion-weighted images in stroke and transient ischemic attack. Stroke. 2008;39(6):1898-900. 
14.Doubal FN, Dennis MS, Wardlaw JM. Characteristics of patients with minor ischaemic strokes and negative MRI: a cross-sectional study. J Neurol Neurosurg Psychiatry. 2011;82(5):540-2.

15. Kawano H, Hirano T, Nakajima M, Inatomi $Y$, Yonehara T. Diffusion-weighted magnetic resonance imaging may underestimate acute ischemic lesions: cautions on neglecting a computed tomography-diffusion-weighted imaging discrepancy. Stroke. 2013;44(4):105661.

16.Kim EY, Ryoo JW, Roh HG, Lee KH, Kim SS, Song IC, et al. Reversed discrepancy between CT and diffusion-weighted MR imaging in acute ischemic stroke. American Journal of Neuroradiology. 2006;27(9):1990-5.

17.Kim JT, Park MS, Kim MK, Cho KH. Minor stroke with total mismatch after acute MCA occlusion. J Neuroimaging. 2011;21(4):399-402.

18. Kim K, Kim BJ, Huh J, Yang SK, Yang MH, Han $\mathrm{M}-\mathrm{K}$, et al. Delayed Lesions on DiffusionWeighted Imaging in Initially Lesion-Negative Stroke Patients. J Stroke. 2021;23(1):69-81.

19.Lovblad KO, Laubach HJ, Baird AE, Curtin F, Schlaug G, Edelman RR, et al. Clinical experience with diffusion-weighted MR in patients with acute stroke. AJNR Am J Neuroradiol. 1998;19(6):1061-6.

20.Oppenheim C, Logak M, Dormont D, Lehericy S, Manai R, Samson Y, et al. Diagnosis of acute ischaemic stroke with fluid-attenuated inversion recovery and diffusion-weighted sequences. Neuroradiology. 2000;42(8):602-7.

21.Chalela JA, Kidwell CS, Nentwich LM, Luby M, Butman JA, Demchuk AM, et al. Magnetic resonance imaging and computed tomography in emergency assessment of patients with suspected acute stroke: a prospective comparison. Lancet. 2007;369(9558):293-8.

22.Simonsen CZ, Madsen MH, Schmitz ML, Mikkelsen IK, Fisher M, Andersen G. Sensitivity of diffusion- and perfusion-weighted imaging for diagnosing acute ischemic stroke is $97.5 \%$. Stroke. 2015;46(1):98-101.

23.Felfeli P, Wenz H, Al-Zghloul M, Groden C, Forster A. Combination of standard axial and thinsection coronal diffusion-weighted imaging facilitates the diagnosis of brainstem infarction. Brain Behav. 2017;7(4):e00666.

24. Warach S, Kidwell CS. The redefinition of TIA: the uses and limitations of DWI in acute ischemic cerebrovascular syndromes. Neurology. 2004;62(3):359-60.
25.Bulut HT, Yildirim A, Ekmekci B, Eskut N, Gunbey HP. False-negative diffusion-weighted imaging in acute stroke and its frequency in anterior and posterior circulation ischemia. $\mathrm{J}$ Comput Assist Tomogr. 2014;38(5):627-33.

26. Oppenheim C, Stanescu R, Dormont D, Crozier S, Marro B, Samson Y, et al. False-negative diffusion-weighted MR findings in acute ischemic stroke. AJNR Am J Neuroradiol. 2000;21(8):1434-40.

27.Cihangiroglu M, Ulug AM, Firat Z, Bayram A, Kovanlikaya A, Kovanlikaya I. High b-value diffusion-weighted MR imaging of normal brain at 3T. Eur J Radiol. 2009;69(3):454-8.

28.Frayne R, Goodyear BG, Dickhoff P, Lauzon ML, Sevick RJ. Magnetic resonance imaging at 3.0 Tesla: challenges and advantages in clinical neurological imaging. Invest Radiol. 2003;38(7):385-402.

29.Benameur K, Bykowski JL, Luby M, Warach S, Latour LL. Higher prevalence of cortical lesions observed in patients with acute stroke using highresolution diffusion-weighted imaging. AJNR Am J Neuroradiol. 2006;27(9):1987-9.

30.Kuhl CK, Textor J, Gieseke J, von Falkenhausen $\mathrm{M}$, Gernert S, Urbach H, et al. Acute and subacute ischemic stroke at high-field-strength (3.0-T) diffusion-weighted MR imaging: intraindividual comparative study. Radiology. 2005;234(2):50916.

31.Zheng Y-q, Li X-m. Comparison of Diagnostic Effects of T2-Weighted Imaging, DWI, SWI, and DTI in Acute Cerebral Infarction. Cardiovascular Innovations and Applications. 2021;5(4):283-7.

32. Kim HJ, Choi CG, Lee DH, Lee JH, Kim SJ, Suh DC. High-b-value diffusion-weighted MR imaging of hyperacute ischemic stroke at 1.5T. AJNR Am J Neuroradiol. 2005;26(2):208-15.

33. Ract I, Ferre JC, Ronziere T, Leray E, CarsinNicol B, Gauvrit JY. Improving detection of ischemic lesions at 3 Tesla with optimized diffusion-weighted magnetic resonance imaging. J Neuroradiol. 2014;41(1):45-51. 